\title{
GROUP ACTIONS ON Q-F-RINGS
}

\author{
J. L. PASCAUD AND J. VALETTE
}

\begin{abstract}
Let $B$ be a ring, $G$ a finite group of automorphisms acting on $B$ and $B^{G}$ the fixed subring of $B$. We give an example of a $B$ which is quasi-Frobenius (Q-F) such that $B^{G}$ is not quasi-Frobenius.
\end{abstract}

S. Jøndrup [3] claims that if card $G$ is a unit in $B$ and if $B$ is self-injective then $B^{G}$ is self-injective. J. Fisher and J. Osterburg [2] use this assertion to prove that if $B$ is quasi-Frobenius then $B^{G}$ is quasi-Frobenius. However we show that this result fails.

Suppose that $A$ is a commutative local artinian ring with Jacobson radical $R$ and call $E$ the injective hull of the simple $A$-module $S=A / R . E$ is finitely generated and faithful [5, théorème 2, p. 97 and corollaire 6, p. 99]. $B$ denotes the ring constructed on the abelian group $A \times E$ with the multiplication

$$
(a, e)\left(a^{\prime}, e^{\prime}\right)=\left(a a^{\prime}, a e^{\prime}+a^{\prime} e\right) \text {. }
$$

LEMMA. $B$ is a commutative local quasi-Frobenius ring.

It is clear that $B$ is commutative local artinian with radical $R \times E$ and that $S^{\prime}=O \times S$ is a simple ideal of $B$ essential in the ideal $O \times E . O \times E$ is essential in $B$, since for each $a \in A-\{o\}$ there exists $e^{\prime} \in E$ such that $\left(o, e^{\prime}\right)(a, e)=\left(o, a e^{\prime}\right)$ is nonzero, because $E$ is faithful. Thus $S^{\prime}$ is essential in $B$.

Then for each $f \in \operatorname{Hom}_{B}\left(S^{\prime}, B\right)-\{o\}, f\left(S^{\prime}\right)$ and $S^{\prime}$ are equal so that $\operatorname{Hom}_{B}\left(S^{\prime}, B\right)$ is isomorphic to $S^{\prime}$. By [1, Theorem 58.6, p. 396], $B$ is quasiFrobenius.

Proposition. (1) There exists a quasi-Frobenius ring $B$ and a finite group $G$ of automorphisms of $B$ with card $G$ invertible in $B$ such that $B^{G}$ is not quasi-Frobenius.

(2) It gives also an example of a quasi-Frobenius ring $C$ with an idempotent $e$ such that eCe is not quasi-Frobenius.

(1) Consider a field $K$ of characteristic different from 2 and define on $K^{3}$ a ring $A$ by the multiplication

$$
(a, b, c)\left(a^{\prime}, b^{\prime}, c^{\prime}\right)=\left(a a^{\prime}, a b^{\prime}+a^{\prime} b, a c^{\prime}+a^{\prime} c\right) .
$$

$A$ is a commutative local artinian ring whose socle has length 2 . Thus $A$ is not quasi-Frobenius.

Received by the editors May 9, 1978 and, in revised form, November 15, 1978.

AMS (MOS) subject classifications (1970). Primary 16A36, 16A72, 16A74.

Key words and phrases. Finite group of automorphisms acting on a ring, quasi-Frobenius ring. 
Define $B$ as before. $(a, e) \mapsto(a,-e)$ is an automorphism of $B$ of order 2 with fixed subring $A$.

(2) As in [2] consider the "twisted" group ring $C$ defined on the set of all formal sums $\sum_{g \in G} b_{g} g$ with $b_{g} \in B$, by the multiplication $g \cdot r=r^{8} g ; e=$ $\left(\Sigma_{g \in G} g\right) /$ card $G$ is an idempotent of $C$ and $e C e$ is isomorphic to $B^{G}=A$ [2]. But it is easy to adapt the classical demonstration of injectivity of group rings [5, pp. 103-104] to prove that $C$ is quasi-Frobenius.

\section{REFERENCES}

1. C. Curtis and J. Reiner, Representation theory of finite groups and associative algebras, Interscience, New York, 1966.

2. J. Fisher and J. Osterburg, Some results on rings with finite group actions, Lecture Notes in Pure and Appl. Math., vol. 25, Dekker, New York, 1977.

3. S. Jondrup, Groups acting on rings, J. London Math. Soc. (2) 8 (1974), 483-486.

4. B. L. Osofsky, A generalization of quasi-Frobenius rings, J. Algebra 4(1966), 373-387.

5. G. Renault, Algèbre non commutative, Gauthier-Villars, Paris, 1975.

Département de Mathématioues, Universtté de Pottiers, 40, Avenue du Rectrur Pineau, 86022 Potriers, France 